\title{
Cross-Layer Resource Allocation for Integrated Voice/Data Traffic in Wireless Cellular Networks
}

\author{
Hai Jiang, Student Member, IEEE, and Weihua Zhuang, Senior Member, IEEE
}

\begin{abstract}
A major task in next-generation wireless cellular networks is provisioning of quality of service $(\mathrm{QoS})$ over the bandwidth limited and error-prone wireless link. In this paper, we propose a cross-layer design scheme to provide QoS for voice and data traffic in wireless cellular networks with differentiated services (DiffServ) backbone. The scheme combines the transport layer protocols and link layer resource allocation to both guarantee the QoS requirements in the transport layer and achieve efficient resource utilization in the link layer. Optimal resource allocation problems for voice and data flows are formulated to guarantee pre-specified QoS with minimal required resources. For integrated voice/data traffic in a cell, a hybrid time-division/code-division medium access control (MAC) scheme is presented to achieve efficient multiplexing. Theoretical analysis and simulation results demonstrate the effectiveness of the proposed cross-layer approach.
\end{abstract}

Index Terms - Code-division multiple access (CDMA), crosslayer design, differentiated services (DiffServ), packet scheduling, quality of service (QoS).

\section{INTRODUCTION}

$\mathbf{I}$ $\mathrm{N}$ THE past decade, the Internet has started to penetrate into the wireless domain. It is now widely recognized that the third generation (and beyond) wireless mobile codedivision multiple access (CDMA) cellular networks are evolving into an all-IP (Internet Protocol) architecture, to provision broadband and seamless global access for various IP multimedia services. A major task in the establishment of such an all-IP platform is provisioning of quality of service (QoS) to different Internet applications.

Recently, the differentiated services (DiffServ) [1] approach has emerged as an efficient and scalable solution to ensure QoS in future IP networks. As a class-based traffic management mechanism, DiffServ does not use per-flow resource reservation and per-flow signaling in core routers, which makes DiffServ scalable. Current research on DiffServ is mainly focused on the wireline network. The bottleneck of such a network is normally assumed to be in the core network. The link from users to the edge router is assumed to have sufficient resources. However, in a hybrid wireless/wireline network, the above assumption does not hold. The bottleneck for an endto-end application across a hybrid wireless/wireline domain is

Manuscript received May 15, 2004; revised December 18, 2004; accepted February 10, 2005. The associate editor coordinating the review of this paper and approving it for publication was E. Hossain. This work was supported by the Premier's Research Excellence Award (PREA) from the Ontario Government and a research grant from the Natural Science and Engineering Research Council (NSERC) of Canada.

The authors are with the Centre for Wireless Communications (CWC), Department of Electrical and Computer Engineering, University of Waterloo, Waterloo, ON N2L 3G1, Canada (e-mail: \{hjiang, wzhuang\}@bbcr.uwaterloo.ca).

Digital Object Identifier 10.1109/TWC.2006.02025.

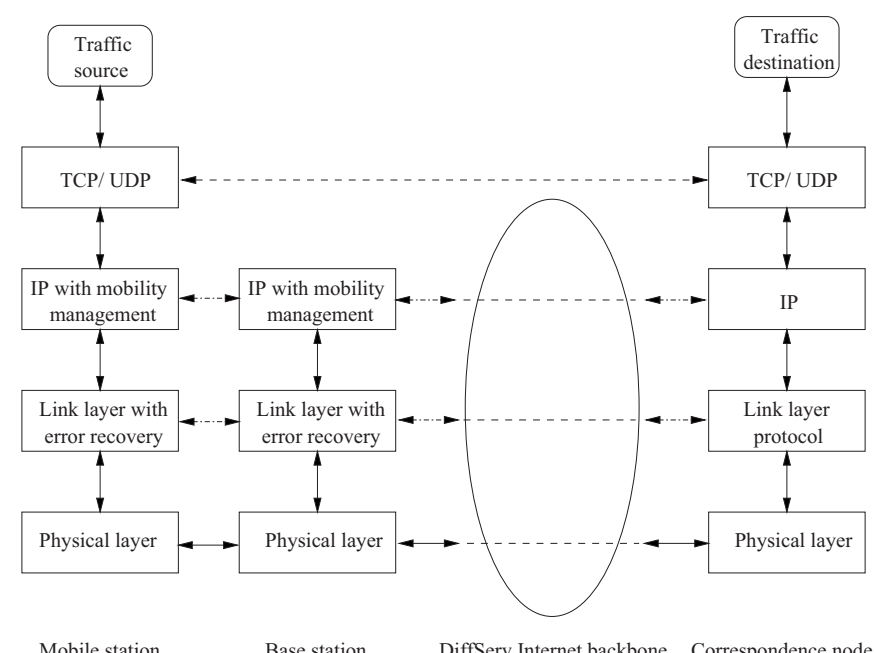

Fig. 1. Protocol stack architecture in the hybrid wireless/wireline IP-based network.

usually the link between the base station (BS) and the mobile station (MS), due to the limited radio resources and the varying characteristics of the radio channel. On the other hand, current medium access control (MAC) schemes [2], [3], [4] in CDMA wireless systems usually provide priority to voice users. Voice traffic flows are scheduled for transmission first, while data traffic flows use the residual system capacity and are not guaranteed with QoS satisfaction, nor are they differentiated from each other. So far, research on QoS support for data traffic is very limited. In [5], [6], two packet-switching scheduling schemes are proposed for wireless CDMA communications. Both are based on per-packet information, thus increasing the scheduling burden and system overhead. Furthermore, the QoS provisioning for data traffic in these two schemes is limited up to the link layer, i.e., only physical layer QoS and link layer QoS are considered. To the best of our knowledge, there is no proposed solution to provide data traffic with higher layer QoS, e.g., transmission rate guarantee at the transport layer, which can be a main concern from the users' point of view.

To address the above issues, in this paper, we propose a cross-layer design scheme for wireless cellular networks with a DiffServ backbone to provide QoS to MSs. The proposed scheme combines the transport layer protocols and link layer resource allocation to both guarantee QoS requirements in the transport layer and achieve efficient resource utilization in the link layer. The rest of this paper is organized as follows. In Section II, the system model is described and an equivalent resource definition is introduced. Sections III- 
TABLE I

SUMMARY OF IMPORTANT SYMBOLS USED.

\begin{tabular}{|c|c|}
\hline Symbol & Definition \\
\hline$C_{d}$ & Estimated available instantaneous resource amount in a frame for all the data flows \\
\hline$C_{i}^{e}$ & Equivalent resource amount for flow $i$ \\
\hline$C^{s}(\cdot, \cdot)$ & Instantaneous resource amount required in a frame by a traffic flow \\
\hline$D_{w e}$ & Sum of delay components (in TCP round trip time) other than wireless queueing delay \\
\hline$D_{w s}$ & Wireless queueing delay of a TCP connection \\
\hline$D_{U}$ & Wireless delay bound for a voice flow in frames \\
\hline$f_{U}$ & UDP packet interarrival time in frames \\
\hline$L_{R}$ & The total time slot number in a frame for voice/data flows in the reverse link \\
\hline$M_{i}$ & Target scheduled link layer (LL) unit number for data flow $i$ \\
\hline$M_{T}$ & Number of LL units that a TCP packet consists of \\
\hline$M_{U}$ & Number of LL units that a UDP packet consists of \\
\hline$m_{i}$ & Instantaneous LL unit number from flow $i$ to be transmitted in a frame \\
\hline$m_{i}^{*}$ & LL unit number of flow $i$ to be actually scheduled in a frame \\
\hline $\max _{t h} / \min _{t h}$ & Maximum/minimum threshold of the RED queue \\
\hline$N_{k}$ & LL unit number remaining at the sender at the beginning of frame $k$ \\
\hline$P_{a}(\cdot)$ & Probability of a TCP packet entering the RED queue \\
\hline$P_{d}(\cdot)$ & Packet dropping probability of the RED queue \\
\hline$P_{e}(\cdot)$ & Bit error probability of an AWGN channel \\
\hline$P_{\max }$ & Maximum dropping probability in the RED queue \\
\hline$P_{s}$ & Probability of successful LL unit transmission \\
\hline$P_{T}$ & TCP packet loss event rate \\
\hline$P_{U}$ & UDP packet loss probability \\
\hline$P_{U, m}$ & The required upper bound of UDP packet loss probability \\
\hline$P_{w e}$ & The packet loss rate of a TCP connection in the wireline domain \\
\hline$P_{w s}$ & The packet loss rate of a TCP connection in the wireless domain \\
\hline$S_{L L}$ & Number of bits in an LL unit \\
\hline$S_{p}$ & Number of payload bits in an LL unit \\
\hline$S_{T}$ & TCP packet size in bits \\
\hline$T_{f}$ & Link layer frame duration \\
\hline$T_{k}$ & LL offset number at frame $k$ for a voice flow \\
\hline$t_{\mathrm{RTO}}$ & TCP retransmission timeout value \\
\hline$V$ & Achieved TCP throughput \\
\hline$V_{\mathrm{CIR}}\left(V_{\mathrm{CIR}, i}\right)$ & Target transmission rate for data service (flow $i$ ) \\
\hline$W\left(W_{i}\right)$ & The sender's congestion window size of a TCP connection (TCP connection $i$ ) \\
\hline$w_{i}^{s} / w_{i}^{l}$ & Short/long - term fairness weight for data flow $i$ \\
\hline $1 / \alpha(1 / \beta)$ & Mean on (off) period of voice traffic \\
\hline$\Gamma_{i}$ & Received bit energy to interference-plus-noise density ratio of flow $i$ \\
\hline$\Gamma_{U}$ & Received bit energy to interference-plus-noise density ratio for voice flow \\
\hline$\varepsilon$ & Maximum number of bit errors that can be corrected by forward error correction \\
\hline$\tau$ & Round trip time of a TCP connection \\
\hline$\tau_{s}\left(\tau_{s, i}\right)$ & Smoothed round trip time estimate of a TCP connection (TCP connection $i$ ) \\
\hline
\end{tabular}

IV present the optimal resource allocation schemes for voice and data flows, respectively. Section V proposes a packetswitching MAC scheduler for integrated voice/data traffic. The system performance is evaluated in Section VI. Section VII concludes this research. As many symbols are used in this paper, Table I summarizes important ones.

\section{System Model}

We consider a hybrid wireless/wireline IP-based network for providing multimedia traffic to MSs, where the Internet backbone is DiffServ based, and the wireless subnet is a wideband time-division/code-division multiple access (TD/CDMA) cellular system with frequency division duplexing (FDD). In the code domain, multi-code CDMA (MC-CDMA) is considered. Here, we focus on the resource management in the reverse link, as resource allocation in the multiple-access reverse link is much more complex than that in the broadcasting forward link. Fig. 1 shows the protocol stack architecture under consideration.

\section{A. Transport Layer Protocols and QoS Requirements}

In the DiffServ core network, packets are aggregately differentiated by different per-hop behaviors: expedited forwarding $(E F)$ [7] which is aimed at providing a connection of low loss, low delay and low jitter; and assured forwarding (AF) [8] to provide a reliable connection with a target transmission rate, referred to as committed information rate (CIR), denoted by $V_{\mathrm{CIR}}$. Generally, in core networks, EF can be applied to voice/video conversation traffic with a stringent delay requirement, while data traffic can be serviced by AF. 


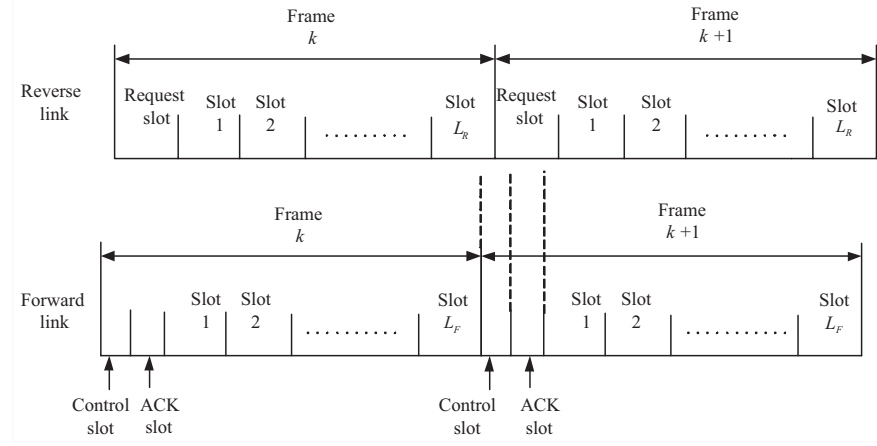

Fig. 2. Time frame structure of the FDD forward and reverse links.

User Datagram Protocol (UDP) is used for voice traffic in our system, which does not use retransmissions to guarantee reliable delivery. UDP itself does not provide mechanisms to ensure timely delivery or other QoS guarantees, but relies on lower layer services to do so. When a voice user is on talk spurt, the UDP packets will be generated periodically. On the other hand, Transmission Control Protocol (TCP) can provide reliable end-to-end transmission over unreliable IP service, which is suitable for the data traffic. Each transport layer (TCP or UDP) packet ${ }^{1}$ is segmented into a number of link layer (LL) units for transmission over the error-prone wireless link, and then reassembled at the BS.

To interwork with the DiffServ backbone, the QoS requirement for voice traffic in the CDMA cellular network is a guaranteed delay bound. If a UDP packet cannot be delivered to the BS within this bound, it will be dropped, and the dropping probability is bounded by $P_{U, m}$ (e.g., $P_{U, m}=1 \%$, a typical value for voice service). The QoS requirement for data service is the guaranteed transport layer throughput $V\left(\geq V_{\mathrm{CIR}}\right)$ with a reliable end-to-end transmission.

\section{B. Hybrid TD/CDMA Architecture at the Link Layer}

At the link layer, we consider a hybrid TD/CDMA architecture, as it has the flexibility in time scheduling and can allow simultaneous transmissions from different MSs via code domain multiplexing. Fig. 2 shows the time frames of the forward and reverse links operating in the FDD mode. Although only the reverse link is considered in this paper, the frame architecture for the forward link is illustrated as acknowledgements (ACKs) for the reverse link are sent from the BS over the forward link. This frame diagram is similar to the one used in [5]. The main difference is: our model ensures that the ACK for any LL unit transmitted in a reverse link frame is received before any packet slot of the next frame. This is important for timely retransmissions in the case of transmission losses.

In the TD/CDMA architecture, time is partitioned into fixed length frames. In the reverse link, each frame is divided into a request slot and $L_{R}$ packet slots; while in the forward

\footnotetext{
${ }^{1}$ In this paper, the terms network "packets" and transport "segments" are used interchangeably for the following reason: For a data connection, the maximum segment size can be negotiated by modern TCP at the connection setup state, thus avoiding IP fragmentation; For a voice connection, the size of a segment is relatively small, thus generally corresponding to one IP packet.
}

link, each frame is divided into a control slot, an ACK slot, and $L_{F}$ packet slots. In each slot of the same type, CDMA multiplexing is used with a fixed spreading gain. An LL unit can be accommodated in a packet slot. When an MS initiates a call, it first issues a request in the request slot based on the slotted ALOHA. The BS responds with the admission decision and slot/code assignment in the control slot of the earliest upcoming forward link frame. The ACK slot in the forward link is used to carry: 1) ACKs for LL units just successfully received in the reverse link; and 2) information of the LL unit number to be scheduled in the next reverse link frame. ACKs for forward link transmissions are piggybacked in the LL units of the reverse link. If a flow is scheduled to transmit several LL units in a frame, these LL units are to be transmitted in the same time slot by MC-CDMA, using mutually orthogonal subcodes generated by multiplying the primary code of the user and the rows of Hadamard-Walsh matrix, i.e., via subcode concatenation [9]. As mutually orthogonal subcodes are used, interference among substreams of a user can be reduced to a large degree, thus increasing the overall system capacity. For multi-code transmission, no extra modifications to the RF part are required, except for higher transmission power. The major additional complexity is in the baseband, which can be addressed by high-speed digital signal processing (DSP) chips [9].

\section{Cross-Layer Design}

In traditional network models, the transport layer and link layer are designed separately and independently. This works well in wireline networks because of the highly reliable transmission provided by the optical fiber channels. However, in a wireless network, for various applications with different QoS requirements, different transport layer protocols have different impacts on lower layers. Hence, an independent link layer resource allocation strategy will not work well. More specifically, for data traffic over a wireless link, TCP dynamically adjusts the sending rate of TCP packets (which will be fed into the link layer transmission queue) according to the network congestion status (e.g., packet loss events and round trip delay); on the other hand, the wireless link layer resource allocation also affects the TCP performance as it ultimately determines the TCP packet loss and transmission delay over the wireless link. That is, the TCP protocol and wireless link layer resource allocation interact with each other. For UDP realtime traffic, it can be seen (in Section III) that the UDP packet arrival pattern and QoS requirement can be incorporated into the link layer design model to achieve an optimal resource utilization. As a result, for link layer resource allocation, a cross-layer design approach is proposed, which takes into account the impact of transport layer protocols and incorporates the transport layer models, in order to meet the high layer QoS requirement and efficiently utilize the scarce resources at the low layer. In addition, when scheduling integrated voice/data traffic, the fairness weights for data traffic at the link layer are calculated based on cross-layer information from the higher layers, to be discussed in Section V. 


\section{Wireless Link Error Recovery}

In a wireless environment, TCP/UDP performance can be degraded severely due to the unreliable link layer transmission. In order to reduce the LL unit loss probability seen by the transport layer, hybrid FEC (forward error correction)/ARQ (automatic repeat request) is used, where ARQ will retransmit the LL units received with noncorrectable bit errors.

Link layer retransmission protocols normally have a constraint on the number of retransmissions, i.e., if an LL unit cannot be received successfully in a given number of retransmissions, the sender will discard this and the subsequent LL units belonging to the same transport layer packet. For a delaysensitive voice flow transmitted with UDP, link layer retransmission limit is jointly determined with the received signal bit energy to interference-plus-noise density ratio $E_{b} / I_{0}$ (referred to as SINR) at the physical layer to guarantee both the delay bound and the packet loss probability bound (to be discussed in Section III). On the other hand, for data traffic, as a TCP packet may consist of many LL units, any discarded LL unit will lead to the loss of the whole TCP packet, thus not utilizing the bandwidth efficiently. So in our system, we do not limit the retransmissions of the LL units for TCP connections. The main concern about unlimited retransmissions is the possible performance degradation due to competing retransmissions between the TCP and the link layer ARQ. TCP Reno and its variants usually employ a coarse granularity of retransmission timeout value (say $500 \mathrm{~ms}$ ). Hence, the impact of competing retransmissions on TCP performance is not significant [10]. Furthermore, in order to control the transmission delay caused by wireless transmissions/retransmissions and possible insufficient wireless resources, the MS employs a random early detection (RED) queue [11] as the TCP packet buffer, because RED has the ability to control the queueing delay and prevent consecutive packet losses. For TCP, consecutive packet losses will trigger timeout more easily than random losses, thus bringing about severe throughput degradation (as the sender does not send packets until a timer, normally with granularity $500 \mathrm{~ms}$, expires). The packet losses in the RED queue will be recovered by TCP to obtain end-to-end reliable transmissions. As a result, the MS will see an end-to-end transmission with no error.

\section{E. Equivalent Resource Amount}

In a CDMA system, due to the non-orthogonal nature of the signals simultaneously transmitted from different users, the system capacity is interference-limited. Let $(m, \Gamma)$ denote the resource vector for each traffic flow in a frame, where $m$ is the number of LL units to be transmitted by MC-CDMA in a slot and $\Gamma$ is the SINR for all the $m$ LL units. We first study the capacity of a slot, i.e., under which constraint flows with different resource vectors may be scheduled to transmit in the same slot. Consider a single-cell environment with perfect power control. There are $N$ flows to be transmitted in a slot without limitation on the maximum transmit power at the MSs. Flow $i(i=1, \ldots, N)$ has resource vector $\left(m_{i}, \Gamma_{i}\right)$. Similar to [12], the received power vector $\mathbf{P}=\left(P_{1}, \ldots, P_{N}\right)\left(P_{i}\right.$ is the received power for an LL unit of flow $i$ ) can be obtained by solving the following linear equations
$\frac{G P_{i}}{\sum_{j \neq i} m_{j} P_{j}+\left(1-\xi_{i}\right)\left(m_{i}-1\right) P_{i}+\eta_{0} W_{r}}=\Gamma_{i}, \quad \forall i=1, \ldots, N$

where $G$ is the processing gain, $W_{r}$ is the total reverse link bandwidth, and $\eta_{0}$ is the one-sided power spectral density of background additive white Gaussian noise. Although for flow $i$ the transmitted $m_{i}$ MC-CDMA substreams use orthogonal subcodes, the orthogonality at the receiver side cannot be kept perfectly, taking into account the transmission delay spread in a multipath propagation environment. Similar to CDMA downlink transmissions with orthogonal codes, an orthogonality factor $\xi_{i} \in[0,1]$ is used to reflect the orthogonality of the $m_{i}$ substreams at the receiver, where $\xi_{i}=1$ means no interference among the $m_{i}$ sub-streams.

After a mathematical manipulation, the received power for an LL unit of flow $i$ can be derived and is given by (2). Thus, the following inequality should hold to make $P_{i}$ positive

$$
\sum_{j=1}^{N} \frac{m_{j} \Gamma_{j}}{G+m_{j} \Gamma_{j}-\left(1-\xi_{j}\right)\left(m_{j}-1\right) \Gamma_{j}}<1 .
$$

From (3), if the total amount of resources in each slot is normalized to 1 , we can define the amount of resources in a slot required by flow $i$ as $C^{s}\left(m_{i}, \Gamma_{i}\right)=\frac{m_{i} \Gamma_{i}}{G+m_{i} \Gamma_{i}-\left(1-\xi_{i}\right)\left(m_{i}-1\right) \Gamma_{i}}$. As in each frame all the LL units from a flow are to be transmitted in one slot, $C^{s}\left(m_{i}, \Gamma_{i}\right)$ is also the resource amount in a frame for flow $i$. For flow $i$, the number of LL units scheduled (i.e., $m_{i}$ ) in different frames generally varies with time. This leads to a variation of the resource amount $C^{s}\left(m_{i}, \Gamma_{i}\right)$ from frame to frame. The average amount of long-term resources required for flow $i$ is defined as $C_{i}^{e}=$ $\sum_{m_{i}} C^{s}\left(m_{i}, \Gamma_{i}\right) \cdot P\left(m_{i}\right)$, where $P\left(m_{i}\right)$ is the probability of $m_{i}$ LL units scheduled in a frame. For flow $i$, if we define $m_{i}$ as the instantaneous $L L$ unit number to be transmitted in a frame, $C^{s}\left(m_{i}, \Gamma_{i}\right)$ is referred to as instantaneous resource amount in this frame, and $C_{i}^{e}$ is referred to as equivalent resource amount. One objective of this research is to guarantee the transport layer QoS with minimal $C_{i}^{e}$.

\section{Voice TRAFFic With DELAY AND PACKET LOSS PROBABILITY BOUNDS}

In the system, voice traffic is characterized by an on/of $\mathrm{f}$ model, where the on and off periods are exponentially distributed with mean values $1 / \alpha$ and $1 / \beta$, respectively. In the on state, the UDP packet interarrival time is $f_{U}$ frames. Each UDP packet is segmented into $M_{U}$ LL units. When a UDP packet is generated, it requires that all the $M_{U}$ LL units be delivered successfully to the BS within $D_{U}$ link layer frames (i.e., wireless delay bound is $D_{U}$ frames); otherwise, the UDP packet is considered useless and will be discarded.

If frame $k$ is the first frame after a UDP packet arrival, frame $k$ is said to have zero LL offset with the UDP packet, and the subsequent $f_{U}-1$ frames have LL offsets ranging from 1 to $f_{U}-1$, respectively. The link layer scheduling algorithm for UDP packets is as follows. In the first frame (with LL offset 0) after a UDP packet arrival, the $M_{U}$ LL units are transmitted in a slot with a target SINR $\Gamma_{U}$ which is to be determined. In each of the subsequent $D_{U}-1$ frames, any 


$$
P_{i}=\eta_{0} W_{r}\left[\frac{G}{\Gamma_{i}}+m_{i}-\left(1-\xi_{i}\right)\left(m_{i}-1\right)\right]^{-1}\left[1-\sum_{j=1}^{N} \frac{m_{j} \Gamma_{j}}{G+m_{j} \Gamma_{j}-\left(1-\xi_{j}\right)\left(m_{j}-1\right) \Gamma_{j}}\right]^{-1}, \quad \forall i=1, \ldots, N
$$

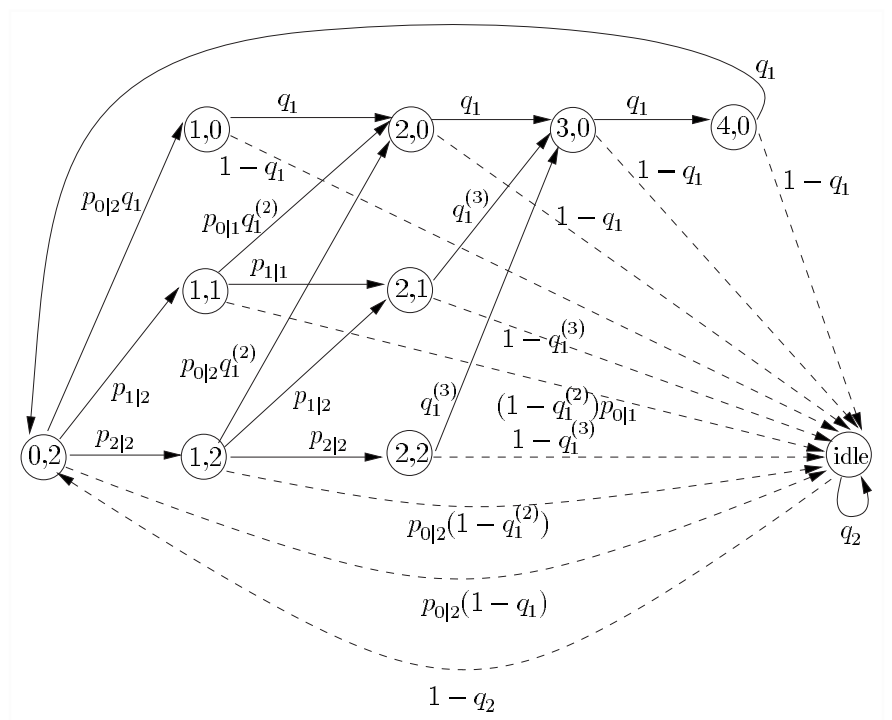

Fig. 3. State transition diagram for a voice flow for $f_{U}=5, M_{U}=2$, and $D_{U}=3$.

LL unit will be retransmitted if not received successfully in the previous frame. If any of the $M_{U}$ LL units cannot be received successfully by the BS within the $D_{U}$ frames, the corresponding UDP packet will be dropped. Let $T_{k}$ denote the LL offset number at frame $k$, and $N_{k}$ the number of LL units remaining in the transmission queue at the beginning of the frame (i.e., $N_{k}$ LL units to be transmitted in this frame). We sample vector $\left(T_{k}, N_{k}\right)$ at the beginning of each frame $k$, and form a discrete-time Markov process with state space $\mathcal{S}=\left\{\left(T_{k}, N_{k}\right), 0 \leq T_{k} \leq f_{U}-1,0 \leq N_{k} \leq M_{U}\right\} \cup\{$ idle $\}$, where the additional idle state represents the voice traffic being in an of $f$ state and no LL units waiting for transmissions at the sender's queue. The state transition diagram is shown in Fig. 3 for $f_{U}=5, M_{U}=2$, and $D_{U}=3$. The transitions between $\left(T_{k}, N_{k}\right)$ states and the idle state are represented by dashed lines, while others by solid lines. $T_{k}$ takes value from 0 to $f_{U}-1$ (i.e., 4 in the case). For the time frame with $T_{k}=0, M_{U}$ (i.e., 2 in the case) LL units from an arrival UDP packet are ready for transmission, thus corresponding to state $(0,2)$. When $T_{k}=1$, there are 3 states $(1,0),(1,1)$, and $(1,2)$, corresponding to the cases when 0,1 , and 2 packets are transmitted unsuccessfully in the previous frame, respectively. Similarly, there are also 3 states for $T_{k}=2$. When $T_{k}=3$, the delay bound is reached. All unsuccessfully transmitted LL units are dropped, therefore there is only one state $(3,0)$. For $T_{k}=4$, as the transmission queue is empty, only one state $(4,0)$ exists. If an on to off transition for the voice traffic occurs between LL offset 0 and $D_{U}-1$ frames (i.e., during LL unit transmissions/retransmissions), the idle state will not be entered until all necessary transmissions or retransmissions are finished.

Consider the probability of a successful LL unit trans- mission (denoted by $P_{s}$ ), i.e., no noncorrectable errors in a received LL unit and thus no need for retransmission. If errors in a received LL unit can be corrected by FEC, this LL unit is successfully received and can be removed from the sender queue system; otherwise, retransmission is needed before the delay bound is violated. For the FEC code, let $S_{L L}$ denote the number of bits in an LL unit, $S_{p}$ the number of payload bits, and $\varepsilon$ the maximum number of bit errors that can be corrected. Then $P_{s}$ is given by

$$
P_{s}=\sum_{j=0}^{\varepsilon}\left(\begin{array}{c}
S_{L L} \\
j
\end{array}\right)\left[P_{e}\left(\Gamma_{U}\right)\right]^{j}\left[1-P_{e}\left(\Gamma_{U}\right)\right]^{S_{L L}-j}
$$

where $P_{e}\left(\Gamma_{U}\right)$ is the probability of bit error for an additive white Gaussian noise (AWGN) channel.

The state transition probabilities shown in Fig. 3 are defined as follows:

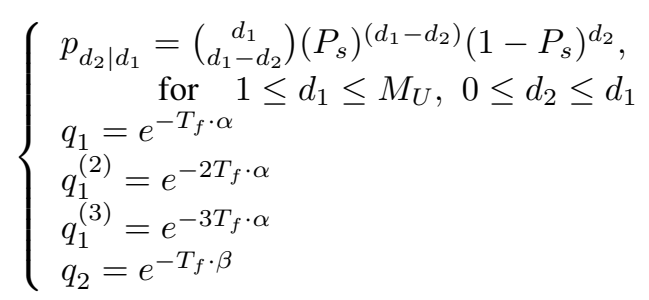

where $T_{f}$ is the link layer frame duration, $p_{d_{2} \mid d_{1}}$ is the conditional probability of $d_{2}$ LL units in the transmission queue at the end of frame $k$ given that there were $d_{1}$ LL units in the queue at the beginning of the frame (i.e., $d_{1}-d_{2}$ LL units are transmitted successfully), $q_{1}\left(q_{2}\right)$ is the conditional probability that the voice remains in on (off) state throughout a frame given than it was in the on (off) state at the beginning of the frame, and $q_{1}^{(2)}\left(q_{1}^{(3)}\right)$ is the conditional probability that the voice remains in the on state from LL offset 0 frame to LL offset 1 ( $L L$ offset 2) frame given that it was on at the beginning of LL offset 0 frame. To understand how the transition probabilities are determined, consider the transition from $(1,1)$ to idle as an example. As the average voice on or off period is usually much larger than the wireless link delay bound, it is reasonable to assume at most one transition between on and of $f$ during the $D_{U}$ possible link layer transmissions for a UDP packet. So a $(1,1)$ to idle transition occurs if both of the following two independent events occur: 1) an on to off transition in LL offset 0 or 1 frame; and 2) the LL unit at the beginning of LL offset 1 frame being transmitted successfully. The probability for the former is $1-q_{1}^{(2)}$, and for the latter is $p_{0 \mid 1}$. Consider transition from $(1,2)$ to $(2,0)$ as another example. It happens if both of the following two independent events occur: 1) the two LL units at the beginning of LL offset 1 frame being transmitted successfully (with probability $p_{0 \mid 2}$ ), and 2) no on to off transition in LL offset 0 or 1 frame (with probability $\left.q_{1}^{(2)}\right)$.

From queue analysis, the steady state probability of each state can be obtained, $P\left(T_{k}, N_{k}\right)$ for state $\left(T_{k}, N_{k}\right)$, and 
$P(i d l e)$ for state idle. As for state $\left(T_{k}, N_{k}\right)$ the instantaneous LL unit number to be transmitted is $N_{k}$, the equivalent resource amount of a voice flow is given by

$$
C_{i}^{e}=\sum_{\left(T_{k}, N_{k}\right) \in \mathcal{S}} C^{s}\left(N_{k}, \Gamma_{U}\right) \cdot P\left(T_{k}, N_{k}\right) .
$$

The UDP packet loss probability is given by $P_{U}=1-$ $\left[\sum_{j=1}^{D_{U}}\left(1-P_{s}\right)^{j-1} P_{s}\right]^{M_{U}}$, where $\sum_{j=1}^{D_{U}}\left(1-P_{s}\right)^{j-1} P_{s}$ is the probability that an LL unit is received successfully in the $D_{U}$ frames.

In order to achieve efficient resource utilization, the equivalent resource amount should be minimized with a constraint of bounded $P_{U}$ (say, $1 \%$ ). Thus an optimization problem can be formulated:

$$
\underset{\Gamma_{U}}{\operatorname{Minimize}} \sum_{\left(T_{k}, N_{k}\right) \in \mathcal{S}} C^{s}\left(N_{k}, \Gamma_{U}\right) \cdot P\left(T_{k}, N_{k}\right)
$$

Subject to $1-\left[\sum_{j=1}^{D_{U}}\left(1-P_{s}\right)^{j-1} P_{s}\right]^{M_{U}}<1 \%$.

The optimal $\Gamma_{U}$ value can be obtained by an exhaustive search.

\section{Data TrafFic with Guaranteed Throughrut}

We consider the connection from a data service MS with a target TCP layer throughput $V_{\mathrm{CIR}}$ to a fixed correspondence node. A TCP packet is segmented into $M_{T}$ LL units prior to the transmission over the wireless link. In each link layer frame, a data flow $i$ can transmit a maximum number, denoted by $M_{i}$, of LL units with SINR $\Gamma_{i}$ by MC-CDMA, i.e., $m_{i} \leq$ $M_{i} . M_{i}$ is called the target scheduled LL unit number for data flow $i$. The design vector for data service is $\left(M_{i}, \Gamma_{i}\right)$, which needs to be optimized.

\section{A. TCP Throughput Estimation}

As the wireless uplink is considered the bottleneck in the round trip connection, for simplicity, we assume that the other parts contribute a total of $D_{w e}$ (including round trip wireline delay, wireless downlink delay, and propagation delay) to the round trip delay and a small packet loss rate $P_{w e}$ for TCP packet transmission. As DiffServ networks are domain-based, at the connection setup state the BS can estimate the $D_{w e}$ and $P_{w e}$ values by the per-domain behaviors [13] in the end-to-end path of the connection.

The TCP throughput can be estimated by [14]

$$
V=\frac{S_{T}}{\tau \sqrt{\frac{2 b P_{T}}{3}}+t_{\mathrm{RTO}}\left[\min \left(1,3 \sqrt{\frac{3 b P_{T}}{8}}\right)\right] P_{T}\left(1+32 P_{T}^{2}\right)}
$$

where $S_{T}$ is the TCP packet size in bits, $\tau$ is the round trip time, $b$ is the number of packets acknowledged by a received ACK (for the transport layer), $P_{T}$ is TCP packet loss event rate, and $t_{\mathrm{RTO}}$ is retransmission timeout value. The round trip time $\tau$ equals $D_{w s}+D_{w e}$, where $D_{w s}$ is the wireless queueing delay for TCP packets. From (6), we can achieve the target TCP throughput (i.e., $V \geq V_{\mathrm{CIR}}$ ) by controlling the queueing performance and the loss event of TCP packets in the wireless link.

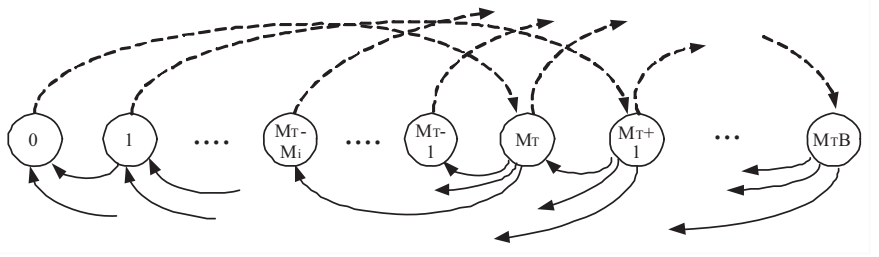

Fig. 4. State transition diagram of the virtual queue for a data flow.

\section{B. Queue Analysis}

There are two buffers at the sender. One is the TCP packet buffer with size $B$ and with RED parameters: minimum threshold $\min _{t h}$, maximum threshold $\max _{t h}$, and maximum dropping probability $P_{\max }$ [11]. The other is the link layer buffer for transmissions and retransmissions of LL units. As a TCP packet is segmented into $M_{T}$ LL units, the two buffers can be viewed as one virtual RED queue which contains LL units with queue size $M_{T} B$ and with service capacity determined by MC-CDMA transmission rate (up to $M_{i}$ LL units to be transmitted in a frame). When a TCP packet arrives and is accepted, the virtual queue content will be increased by $M_{T}$; and when a number of LL units are transmitted successfully by MC-CDMA, the virtual queue occupancy will be reduced by this number. At the beginning of frame $k$, there are $N_{k}$ LL units remaining in the virtual queue. Thus, $\left\{N_{k}, k>0\right\}$ forms a discrete-time Markov process with state space $\left\{0,1, \ldots, M_{T} B\right\}$. The state transition diagram is shown in Fig. 4. A dashed line represents a TCP packet (and correspondingly, $M_{T}$ LL units) entering the queue, and a solid line represents one or more (up to $\min \left(N_{k}, M_{i}\right)$ ) LL units being transmitted successfully.

First, consider the arrival process of the queue system. As in [15], we model the arrival process of TCP packets at the wireless link as a Bernoulli process. For every frame, the probability of a TCP packet arrival is given by $\left(V \cdot T_{f} / S_{T}\right)$. Some TCP packets may be dropped by RED and do not affect the queue evolution. From the virtual queue's point of view, at frame $k$, the probability of $M_{T}$ LL units entering the virtual queue is $P_{a}\left(N_{k}\right)=\left(V \cdot T_{f} / S_{T}\right)\left[1-P_{d}\left(N_{k}\right)\right]$, where the dropping probability is given in (7) with $\lceil\cdot\rceil$ being the ceiling function.

Next, consider the departure process of the virtual queue system. The probability of a successful LL unit transmission is given by (4) with $P_{e}\left(\Gamma_{i}\right)$. In the $k$ th frame, $\min \left(N_{k}, M_{i}\right) \mathrm{LL}$ units are transmitted, i.e., $m_{i}=\min \left(N_{k}, M_{i}\right)$. The probability of $j\left(0 \leq j \leq \min \left(N_{k}, M_{i}\right)\right)$ LL unit departures in the $k$ th frame is

$$
\left(\begin{array}{c}
\min \left(N_{k}, M_{i}\right) \\
j
\end{array}\right)\left(P_{s}\right)^{j}\left(1-P_{s}\right)^{\min \left(N_{k}, M_{i}\right)-j} .
$$

Based on the traffic arrival and departure processes, we can derive the state transition probability matrix of the discretetime Markov process and further obtain the steady state queue distribution $\left[\pi(0), \pi(1), \ldots, \pi\left(M_{T} B\right)\right]$. In the following, based on this distribution, the packet loss rate and delay are calculated, from the TCP packets' point of view.

The TCP packet loss rate over the wireless link is then $P_{w s}=\sum_{j=0}^{M_{T} B} P_{d}(j) \cdot \pi(j)$. In our system, an RED queue is 


$$
P_{d}\left(N_{k}\right)= \begin{cases}0, & N_{k}<M_{T} \cdot \min _{t h} \\ \frac{P_{\max } \cdot\left(\left\lceil\frac{N_{k}}{M_{T}}\right\rceil-\min _{t h}\right)}{\max _{t h}-\min _{t h}}, & M_{T} \cdot \min _{t h} \leq N_{k}<M_{T} \cdot \max _{t h} \\ 1, & N_{k} \geq M_{T} \cdot \max _{t h}\end{cases}
$$

used in the wireless link, which is usually used for data traffic in DiffServ core networks. As RED can prevent consecutive packet losses, the end-to-end TCP packet loss event rate $P_{T}$ can be approximated by $P_{T}=1-\left(1-P_{w s}\right)\left(1-P_{w e}\right)$. The average occupancy of the TCP packet queue is $E\left(q_{T}\right)=$ $\sum_{j=0}^{M_{T} B}\left\lceil\frac{j}{M_{T}}\right\rceil \cdot \pi(j)$. Applying the Little's law, we obtain the mean queueing delay for TCP packets in the wireless link $E\left(D_{w s}\right)=\frac{E\left(q_{T}\right)}{V \cdot\left(1-P_{w s}\right) / S_{T}}$.

In TCP, the retransmission timeout value $t_{\mathrm{RTO}}$ is estimated at the sender by $t_{\mathrm{RTO}}=\tau_{s}+4 \cdot V(\tau)$, where $\tau_{s}$ is the smoothed $\tau$ estimate and $V(\tau)$ is the smoothed estimate of deviation of $\tau$ [16]. From (6), we can see that $t_{\mathrm{RTO}}$ does not affect much the achieved throughput of TCP if the TCP packet loss event rate is small. Here, the simple empirical heuristic of $t_{\mathrm{RTO}} \approx 4 \tau \approx 4\left[D_{w e}+E\left(D_{w s}\right)\right]$ is used as it works reasonably well in the TCP throughput estimation [16].

To achieve the required CIR, based on (6), the following equations should hold

$$
\left\{\begin{array}{l}
V=\frac{S_{T}}{\left[D_{w e}+E\left(D_{w s}\right)\right] \sqrt{\frac{2 b P_{T}}{3}}+t_{\text {RTO }}\left[\min \left(1,3 \sqrt{\frac{3 b P_{T}}{8}}\right)\right] P_{T}\left(1+32 P_{T}^{2}\right)} \\
V \geq V_{\text {CIR. }}
\end{array}\right.
$$

It can be seen that the values of $P_{T}$ and $E\left(D_{w s}\right)$ depend on the design vector $\left(M_{i}, \Gamma_{i}\right)$ of the wireless link. For each possible $M_{i}$ value, the corresponding $\Gamma_{i}$ that makes (8) hold is determined numerically. Such an $\left(M_{i}, \Gamma_{i}\right)$ vector is called a feasible design vector. The equivalent resource amount for this feasible design vector is $C_{i}^{e}=\sum_{j=0}^{M_{T} B} C^{s}\left(\min \left(j, M_{i}\right), \Gamma_{i}\right)$. $\pi(j)$. In order to achieve the target TCP throughput with the minimum required resource amount, we should minimize $C_{i}^{e}$ over the feasible design vectors. Thus the optimization problem is formulated as

$$
\underset{\left(M_{i}, \Gamma_{i}\right)}{\operatorname{Minimize}} \sum_{j=0}^{M_{T} B} C^{s}\left(\min \left(j, M_{i}\right), \Gamma_{i}\right) \cdot \pi(j)
$$

Subject to (8).

The optimal $\left(M_{i}, \Gamma_{i}\right)$ can be found via an exhaustive search among all the feasible design vectors.

\section{MAC PACKet SCHEDUling Algorithm FOR INTEGRATED VOICE/DATA TRAFFIC}

In the preceding sections, we discuss the optimization of the equivalent resource amount for a voice flow and for a data flow, respectively. When different flows are multiplexed together, the resource requirements of all the flows may not be met in each frame. Due to the dynamic nature of the multiplexed traffic, the target scheduled LL unit number $M_{i}$ for data flow $i$ or $M_{U}$ for voice flows may not be affordable in each frame. Thus an efficient MAC scheduler is essential to schedule LL units from these flows in a fair way.

Similar to the case in previous research, voice flows are given priority in the proposed scheduler. In each frame, the scheduler guarantees to transmit all the LL units remaining in each voice flow's transmission queue, including both newly arrived LL units and those waiting for retransmissions. This can be supported by an appropriate call admission control strategy. Data flows share the residual resources in each frame. To implement such a scheduler, resource allocation order is essential. We lose efficiency if we first schedule all the voice LL units and then the data LL units, as generally more resources are required by data flows in each frame. Intuitively, in each frame we should schedule LL units from the flows in the descending order of the required instantaneous resource amount. Hence, usually data LL units are scheduled first. However, if not all the LL units from the voice flows can be scheduled subsequently, we should determine a new feasible instantaneous number $m_{i}^{*}$ of LL units to be actually transmitted for each data flow $i$, and in future frames try to compensate the data flow which experiences performance degradation. Therefore, although data flows may be scheduled first in each scheduling attempt, voice flows have a higher priority.

In frame $k$, for each data flow $i$, the BS has the information of: 1) the optimal $\Gamma_{i}$ value (from Section IV), and 2) the instantaneous LL unit number $m_{i}=\min \left(N_{k}, M_{i}\right)$ to be transmitted. The following weight is used for data flow $i$ in sharing the resources in the frame: $w_{i}=C^{s}\left(m_{i}, \Gamma_{i}\right) \cdot w_{i}^{l} \cdot w_{i}^{s}$, where $w_{i}^{l}$ and $w_{i}^{s}$ are termed as long-term fairness weight and short-term fairness weight, respectively.

Long-term fairness weight - In DiffServ core networks, a data flow $i$ will be serviced by AF with CIR requirement $V_{\mathrm{CIR}, i}$. There is a marker at the BS (i.e., the edge router) to classify the incoming traffic from each flow. The BS measures the transmission rate of flow $i$ during a relatively long period and marks the arrival packets to in-profile or outof-profile based on the comparison of the measured rate $r_{i}$ and the required CIR $V_{\mathrm{CIR}, i}$. Here we choose the long-term fairness weight $w_{i}^{l}=V_{\mathrm{CIR}, i} / r_{i}$. Obviously, as $r_{i}$ decreases from the CIR value, this weight increases. It can be seen that the long-term fairness weight takes advantage of cross-layer information from the network layer.

Short-term fairness weight - A data flow uses TCP as the transport layer protocol. TCP avoids network congestion through controlling the sender's congestion window, $W$, which is an estimated number of TCP packets that can be in transit between the sender and destination without causing congestion. More precisely, the source sends TCP packets until the number of unacknowledged packets reaches $W$ and then uses ACK-clocking to send new packets. The short term transmission rate of TCP flow $i$ can be represented by the ratio of instantaneous TCP congestion window $\left(W_{i}\right)$ times TCP packet size $\left(S_{T}\right)$ to the measured smoothed TCP round trip time $\left(\tau_{s, i}\right)$ at the TCP sender. Here we choose the short-term fairness weight to be $w_{i}^{s}=V_{\mathrm{CIR}, i} /\left[W_{i} \cdot S_{T} / \tau_{s, i}\right]$. Obviously, the short-term fairness weight is calculated based on cross- 
TABLE II

THE ANALYSIS AND SIMULATION PARAMETERS.

\begin{tabular}{c|c||c|c||c|c||c|c}
\hline symbol & value & symbol & value & symbol & value & symbol & value \\
\hline \hline $1 / \alpha$ & $0.352 \mathrm{~s}$ & $1 / \beta$ & $0.650 \mathrm{~s}$ & $S_{L L}$ & 224 bits & $S_{p}$ & 192 bits \\
\hline$\varepsilon$ & 4 bits & $D_{w e}$ & $200 / 40 \mathrm{~ms}$ & $f_{U}$ & 5 frames & $M_{U}$ & 6 LL units \\
\hline$D_{U}$ & 3 frames & $M_{T}$ & 42 LL units & $S_{T}$ & 8000 bits & $T_{f}$ & $10 \mathrm{~ms}$ \\
\hline $\max _{t h}$ & 15 packets & $\min _{t h}$ & 5 packets & $P_{\max }$ & 0.1 & $\xi_{i}$ & 1 \\
\hline
\end{tabular}

layer information from the transport layer.

The total instantaneous resource amount in each frame is $L_{R}$ as there are $L_{R}$ time slots, and the instantaneous resource amount in each slot is normalized to 1 . Let $C_{v}$ denote the sum of the instantaneous resource amounts of all the voice flows in a frame, which is to be guaranteed. The total available instantaneous resource amount in the frame for all the data flows is given as $C_{d}=L_{R}-C_{v}(\geq 0)$. Thus, the estimated instantaneous resource amount that data flow $i$ can use in this frame is $w_{i} \cdot C_{d} /\left[\sum_{j=1}^{N_{d}} w_{j}\right]$, where $N_{d}$ is the number of the total data flows. Based on this amount, we can estimate the number of LL units $m_{i}^{*}$ from flow $i$ which can be actually scheduled in this frame with SINR $\Gamma_{i}$ :

$$
\begin{aligned}
& m_{i}^{*}=\min \left(s, 2 M_{i}\right) \\
& s=\text { maximum integer } n: C^{s}\left(n, \Gamma_{i}\right) \leq w_{i} \cdot C_{d} /\left[\sum_{j=1}^{N_{d}} w_{j}\right] .
\end{aligned}
$$

To avoid over-consumption of the resources and starvation of background traffic, an upper bound is set for $m_{i}^{*}$. Here, the value of $2 M_{i}$ is used as an example. For the simplicity of presentation, in the following, for each voice or data flow $i$ in a frame, $m_{i}^{*}$ denotes the estimated number of LL units to be actually scheduled $\left(m_{i}^{*}=m_{i}\right.$ for voice flow), $C_{i}=C^{s}\left(m_{i}^{*}, \Gamma_{i}\right)$ is the instantaneous resource amount corresponding to $m_{i}^{*}$. The required $\Gamma_{i}$ value $\left(\Gamma_{i}=\Gamma_{U}\right.$ for voice flows) does not change with frames. $\left(m_{i}^{*}, \Gamma_{i}, C_{i}\right)$ is called the instantaneous resource requirement vector of flow $i$ in the frame, to be determined as follows.

At the beginning of the scheduling in each frame, all flows are put into a wait-for-scheduling (WFS) queue to be ready for transmission. Let $S_{l}$ denote the remaining instantaneous resource amount for slot $l\left(l=1,2, \ldots, L_{R}\right)$ after some LL units have been scheduled. Fig. 5 shows the procedure to schedule LL units from $N$ voice/data flows in each frame. If not all the $m_{i}^{*}(i=1,2, \ldots, N)$ LL units from all the flows can be transmitted in the frame, we reduce the estimated available instantaneous resource amount for all the $N_{d}$ data flows from $C_{d}$ to $C_{d} \cdot(1-\delta)$, where $\delta(>0)$ is a small value. If all the $m_{i}^{*}(i=1,2, . ., N)$ LL units of all the flows are scheduled successfully, effort is still needed to schedule extra data service LL units in this frame using the remaining instantaneous resource amount, based on the long-term fairness weights of the data flows (in descending order).

\section{Vi. Performance Evaluation}

Simulations are carried out to evaluate the performance of our proposed approaches, based on event-driven programs coded by $\mathrm{C} / \mathrm{C}++$. A centralized scheduler module is used to allocate resources to voice/data users in each link layer frame.

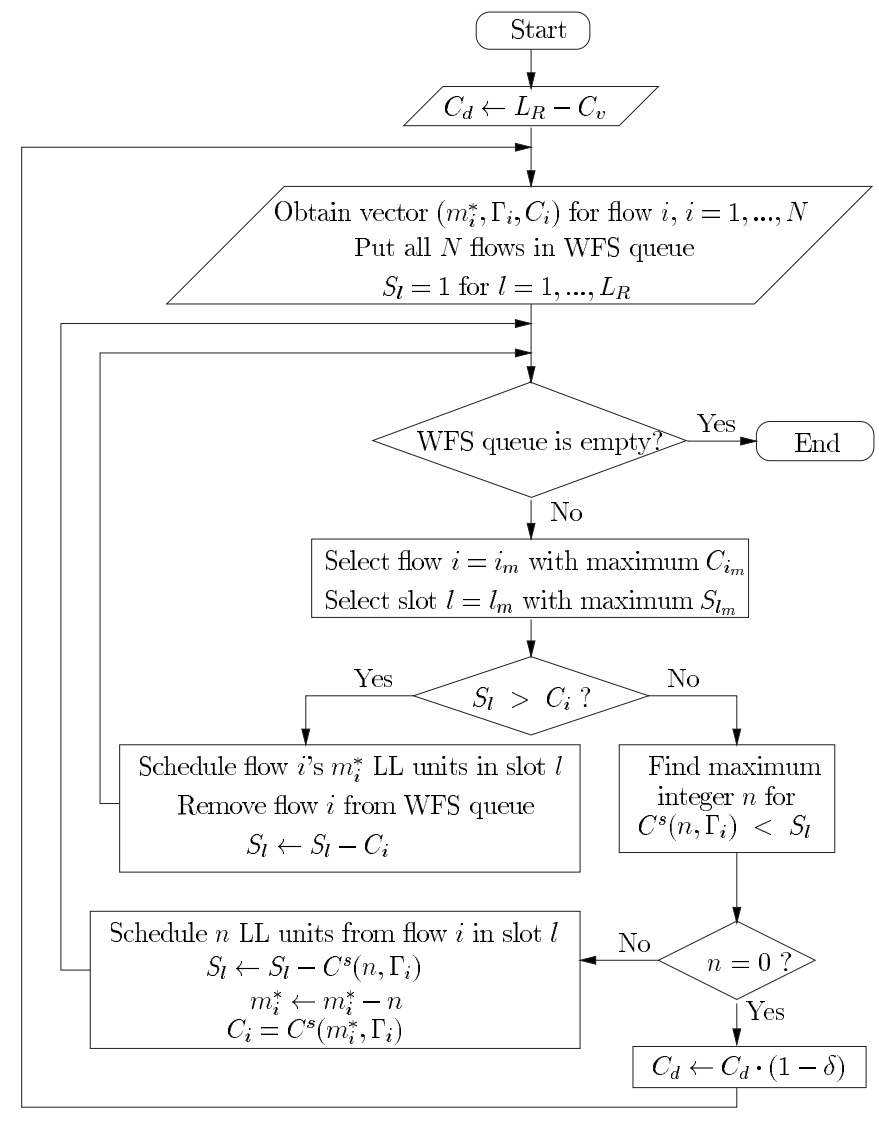

Fig. 5. The proposed MAC scheduling scheme in each frame.

Voice traffic arrivals are based on the on/off model, while data traffic arrivals are managed by the TCP Reno flow control mechanism. An event control module is employed to deal with the events in the system, e.g., packet generation, timeout, etc. As perfect power control is assumed, the wireless channel is simulated by an error rate $P_{e}(\Gamma)$ for a transmitted bit. For MC-CDMA, we consider the case with no interference among substreams from the same user, simulated by an orthogonality factor equal to one. In the physical layer, as an example, we consider differential binary phase shift keying (DBPSK). The probability of bit error as a function of SINR $\Gamma$ for an AWGN channel is given by $P_{e}(\Gamma)=\frac{1}{2} e^{-\Gamma}$. In the link layer, we adopt Bose Chaudhuri Hocquenghem $(\mathrm{BCH})$ code capable of correcting $\varepsilon$ errors. Cyclic redundancy check (CRC) is employed to detect the undetectable errors by the $\mathrm{BCH}$ code. We treat the CRC code as part of the payload of BCH code and assume that the CRC overhead is negligible. Table II lists the parameter values used in the analysis and simulation. Note 


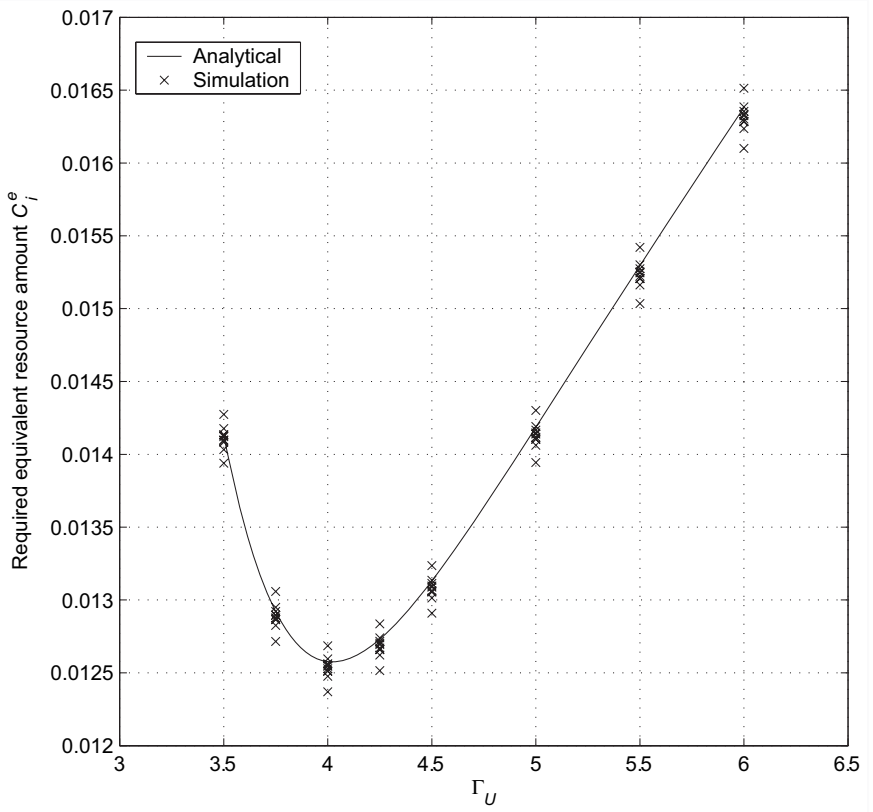

Fig. 6. Equivalent resource amount $C_{i}^{e}$ of a voice flow versus $\Gamma_{U}$ for $f_{U}=5, M_{U}=6$, and $D_{U}=3$.

that the DBPSK and BCH codes are used in this research for illustration. This work can also be applied to other modulation and channel coding schemes such as those used in WCDMA or CDMA2000.

\section{A. Test for a Single Voice Flow}

Consider a single voice flow initiated from an MS. According to the scheduling policy, at each frame, any LL units remaining in the transmission queue are guaranteed to be transmitted. For different values of $\Gamma_{U}$, the equivalent resource amount $C_{i}^{e}$ and the UDP packet loss probability are calculated analytically, and are shown in Fig. 6 and Fig. 7, respectively, for case $f_{U}=5, M_{U}=6$, and $D_{U}=3$. Simulations are also carried out to verify the equivalent resource amount and UDP packet loss probability at the selected $\Gamma_{U}$ values. Ten sampled simulation results for each selected $\Gamma_{U}$ value are shown in Fig. 6 and Fig. 7. It can be seen that the simulation results match well with the analytical results, thus validating the analytical model. Intuitively, when the $\Gamma_{U}$ value is small, the probability of successful transmission is low, thus more retransmissions (and therefore more resources) are required, and the probability of delay bound violation is high. On the other hand, when the $\Gamma_{U}$ value is large, the probability of successful transmission is high, probability of delay bound violation is low, but large transmission power (and therefore more resources) is required to achieve high $\Gamma_{U}$. Therefore, it is expected that an optimal point exists having an intermediate value of $\Gamma_{U}$. It is also observed from Fig. 6 and Fig. 7 that, when $\Gamma_{U}$ is chosen to be in the neighborhood of 4 , the required equivalent resource amount reaches its minimal value of 0.0126 , and the corresponding UDP packet loss probability is approximately $0.1 \%$, well below the target packet loss probability bound $1 \%$.

Among all the successfully transmitted UDP packets, the transmission delay (with unit of link layer frame) takes value

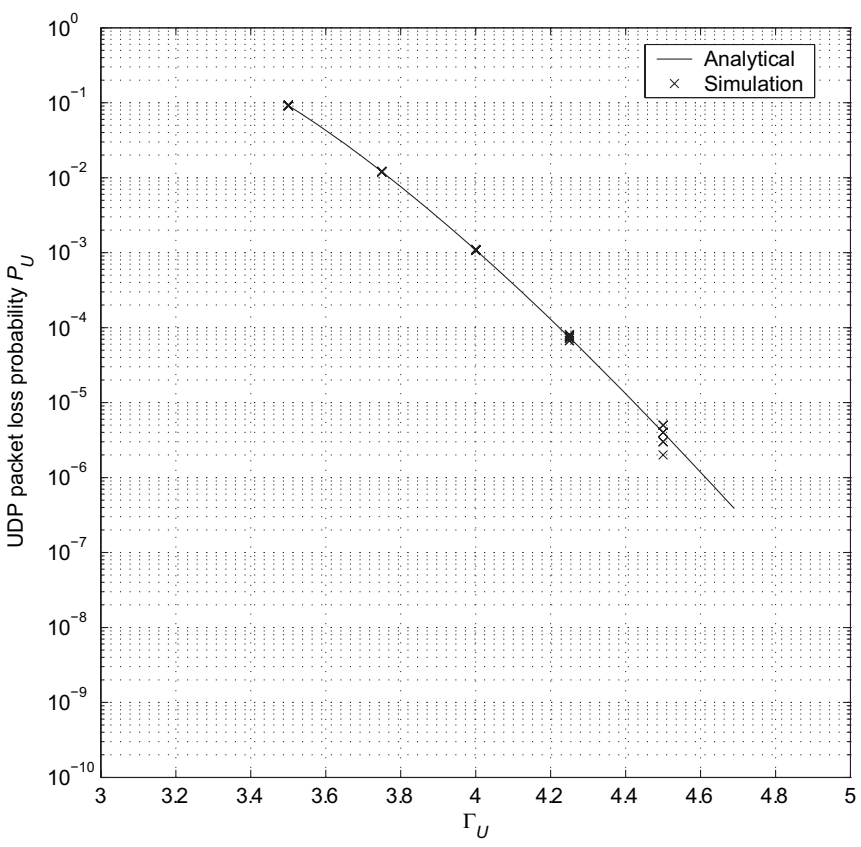

Fig. 7. UDP packet loss probability $P_{U}$ of a voice flow versus $\Gamma_{U}$ for $f_{U}=5, M_{U}=6$, and $D_{U}=3$.

TABLE III

DISTRIBUTION OF UDP PACKET TRANSMISSION DELAY OVER WIRELESS LiNK With $f_{U}=5, M_{U}=6, D_{U}=3$ AND OPTIMUM $\Gamma_{U}$ VALUe 4.02 .

\begin{tabular}{c|c|c|c|c}
\hline \multicolumn{2}{c|}{ Delay (unit: link layer frame) } & 1 & 2 & 3 \\
\hline \hline \multirow{2}{*}{ Probability } & Analysis & $72.28 \%$ & $26.15 \%$ & $1.57 \%$ \\
\cline { 2 - 5 } & Simulation & $72.24 \%$ & $26.16 \%$ & $1.60 \%$ \\
\hline
\end{tabular}

from 1 to $D_{U}$. Table III shows the analytical and simulated results of the distribution of the UDP packet transmission delay over wireless link with the optimum $\Gamma_{U}$ value 4.02 . It can be seen that majority (approximately 70\%) of the UDP packets are transmitted successfully in one link layer frame.

\section{B. Test for a Single Data Flow}

Consider a single data flow initiated from an MS with target throughput $V_{\mathrm{CIR}}=250 \mathrm{~kb} / \mathrm{s}$ and other parameters $D_{w e}=40$ $\mathrm{ms}$ and $P_{w e}=1 \%$. According to the scheduling policy, at each frame, up to $M_{i}$ LL units are scheduled for transmission over the wireless channel by MC-CDMA.

Fig. 8 and Fig. 9 show the achieved TCP throughput and required equivalent resource amount for different feasible design vectors $\left(M_{i}, \Gamma_{i}\right)$ from theoretical analysis and simulation, respectively. As the feasible design vector has one degree of freedom, only values of $M_{i}$ are shown in the horizontal axes of Fig. 8 and Fig. 9. For each selected feasible design vector, 10 independent simulations are carried out. Each simulation runs for 30,000 frames, and the statistics are collected in the last 24,000 frames. In the simulations, the achieved throughput is traced through the accumulative ACKs for TCP packets from the correspondence node.

It can be seen that the simulation results match to the theoretical analysis. Each feasible design vector can guarantee 


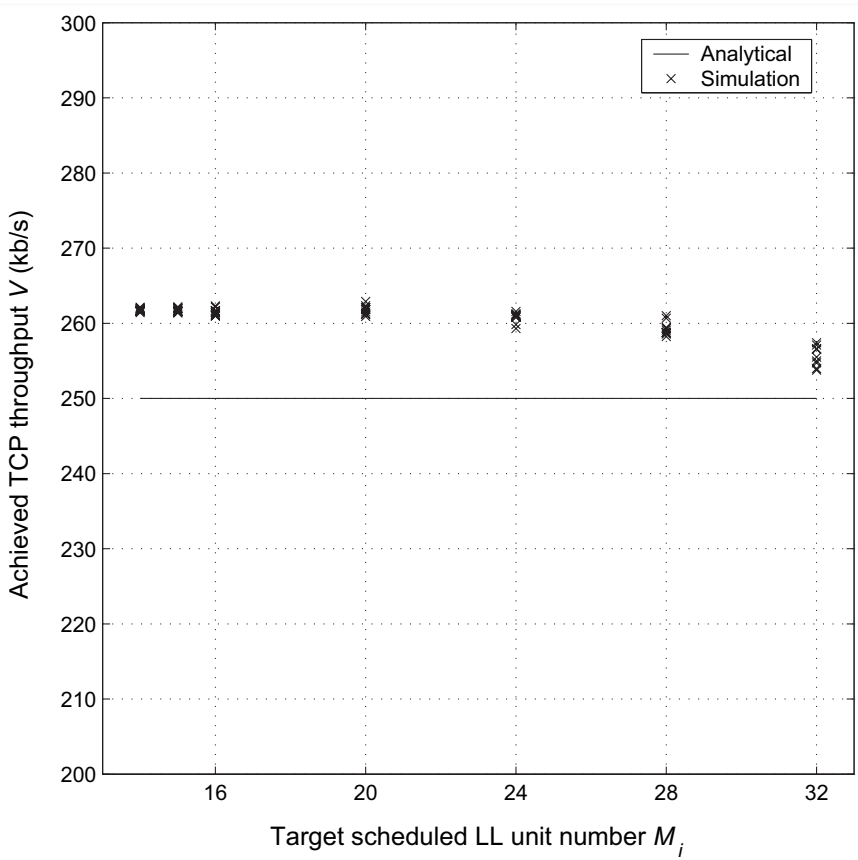

Fig. 8. Achieved TCP throughput $V$ versus $M_{i}$ for a data flow with $V_{\mathrm{CIR}}=$ $250 \mathrm{~kb} / \mathrm{s}$.

the throughput requirement of a TCP flow. In each simulation, the achieved TCP throughput is a bit higher than the required $V_{\mathrm{CIR}}$, thus making the simulated equivalent resource amount a bit higher than the analyzed one. An optimal point $\left(M_{i}=\right.$ 15 , and $\Gamma_{i}=5.48$ in this example) can be obtained among the feasible design vectors to minimize the required equivalent resource amount, thus achieving optimal resource allocation.

\section{Test for Integrated Voice/Data Traffic}

Consider the reverse link transmissions of integrated voice/data traffic in a cell. The UDP transmissions of voice flows and TCP transmissions of data flows are simulated with the proposed packet-switching MAC scheduler. In the link layer, the frame duration is $10 \mathrm{~ms}$, which includes 8 packet slots $\left(L_{R}=8\right)$. For voice traffic, the optimal $\Gamma_{i}$ value $(=4.02)$ and the equivalent resource amount $(=0.0126)$, as shown in Fig. 6, are used. Two types of data traffic are considered with target throughput requirements $V_{\mathrm{CIR}, A}=50 \mathrm{~kb} / \mathrm{s}$ (referred to as type A) and $V_{\mathrm{CIR}, B}=250 \mathrm{~kb} / \mathrm{s}$ (referred to as type B), respectively. As shown in Table IV, 10 long-lived TCP flows (with IDs ranging from 1 to 10) for each type are simulated, 5 (with odd ID numbers) of them have $D_{w e}=$ $200 \mathrm{~ms}$ and the other 5 (with even ID numbers) have $D_{w e}=$ $40 \mathrm{~ms}$. All the TCP flows have $P_{w e}=1 \%$. Each TCP flow is running on a link layer with unlimited retransmissions. Via theoretical analysis for single users, the optimal design vector $\left(M_{i}, \Gamma_{i}\right)$ is $(3,5.61),(3,5.50),(16,5.50)$, and $(15,5.48)$ for the four sub-types, respectively. The corresponding equivalent resource $C_{i}^{e}$ values are given in Table IV. It can be seen that the sum of equivalent resource amounts for all the 20 data flows is 4.742 . In addition to the 20 data flows, we consider 3 different traffic load cases with 300, 258, and 200 voice flows, respectively. In the 3 cases, the sum of the required equivalent resource amounts of all the data/voice flows is 8.5, 8.0, and

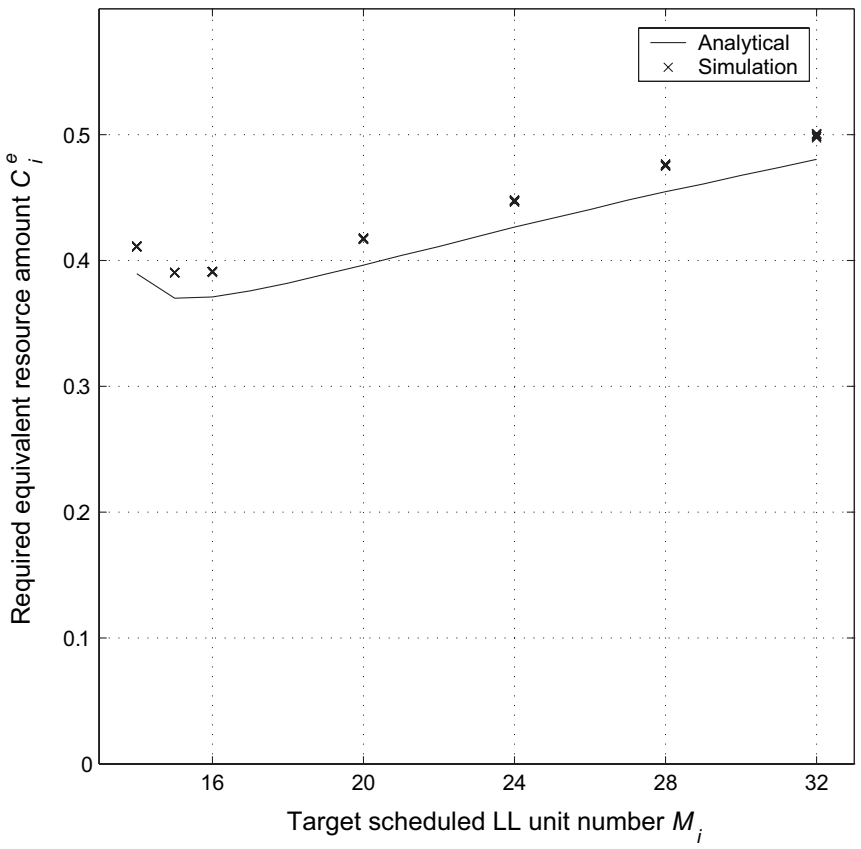

Fig. 9. Required equivalent resource amount $C_{i}^{e}$ versus $M_{i}$ for a data flow with $V_{\mathrm{CIR}}=250 \mathrm{~kb} / \mathrm{s}$.

7.3 , respectively. As the available resource amount is 8 , we terms the 3 cases as under-provisioned (UP), near-provisioned $(N P)$, and over-provisioned $(O P)$ case, respectively.

The simulation for each case runs for 30,000 frames, and the statistics are collected in the last 24,000 frames. During all the simulations, it is observed that the delay bound and packet loss probability requirements for voice flows are guaranteed, as they are given priority in each frame. Fig. 10 shows the achieved throughput for data flows (type A 1-10 and type B 1-10) in the UP, NP, and OP cases. It can be seen that each data flow achieves a throughput around its target value in NP, and this throughput increases (decreases ) in OP (UP). All small throughput flows (type A) achieve almost the same performance, while the performance for large throughput flows (type B) fluctuates in each case where flows with $D_{w e}=40$ ms get a higher throughput than flows with $D_{w e}=200 \mathrm{~ms}$. This is consistent with the well-known observation in previous research: the networks have a bias against TCP flows with a large round trip time and a large target throughput [17]. When both round trip time and target throughput are large (e.g., type B traffic with $D_{w e}=200 \mathrm{~ms}$ ), it takes more time for the TCP window to reach the steady state after reacting to packet losses, thus achieving a relatively small throughput. The above fluctuation (within 5\%) should be acceptable, taking into account the fact that data traffic will be serviced by AF in the DiffServ core network, and AF is intended to provide a coarse QoS assurance level. Thus, the proposed system model can provision QoS satisfactorily, and the proposed MAC scheduling scheme can achieve effectiveness. In addition, as all the data flows experience approximately the same level of throughput loss/gain when under-provisioned/over-provisioned, reasonable fairness in a coarse sense is achieved. This work should also provide helpful insights for call admission control in wireless cellular networks. 
TABLE IV

DATA TRAFFIC PARAMETERS USED IN THE SIMULATIONS FOR INTEGRATED VOICE/DATA TRAFFIC.

\begin{tabular}{|c|c|c|c|c|c|c|c|}
\hline \multirow{2}{*}{ Traffic type } & \multirow{2}{*}{$V_{\mathrm{CIR}}$} & \multirow{2}{*}{$\begin{array}{l}\text { Number } \\
\text { of flows }\end{array}$} & \multirow{2}{*}{$D_{w e}$} & \multirow{2}{*}{$P_{w e}$} & \multicolumn{3}{|c|}{ Optimal point } \\
\hline & & & & & $M_{i}$ & $\Gamma_{i}$ & $C_{i}^{e}$ \\
\hline \multirow{2}{*}{ A } & \multirow{2}{*}{$50 \mathrm{~kb} / \mathrm{s}$} & \multirow{2}{*}{$\begin{array}{c}10 \\
\text { (ID: } 1-10)\end{array}$} & $200 \mathrm{~ms}$ & $1 \%$ & 3 & 5.61 & 0.1076 \\
\hline & & & $40 \mathrm{~ms}$ & $1 \%$ & 3 & 5.50 & 0.1069 \\
\hline \multirow{2}{*}{ B } & \multirow{2}{*}{$250 \mathrm{~kb} / \mathrm{s}$} & \multirow{2}{*}{$\begin{array}{c}10 \\
\text { (ID: } 1-10)\end{array}$} & $200 \mathrm{~ms}$ & $1 \%$ & 16 & 5.50 & 0.3639 \\
\hline & & & $40 \mathrm{~ms}$ & $1 \%$ & 15 & 5.48 & 0.3700 \\
\hline
\end{tabular}

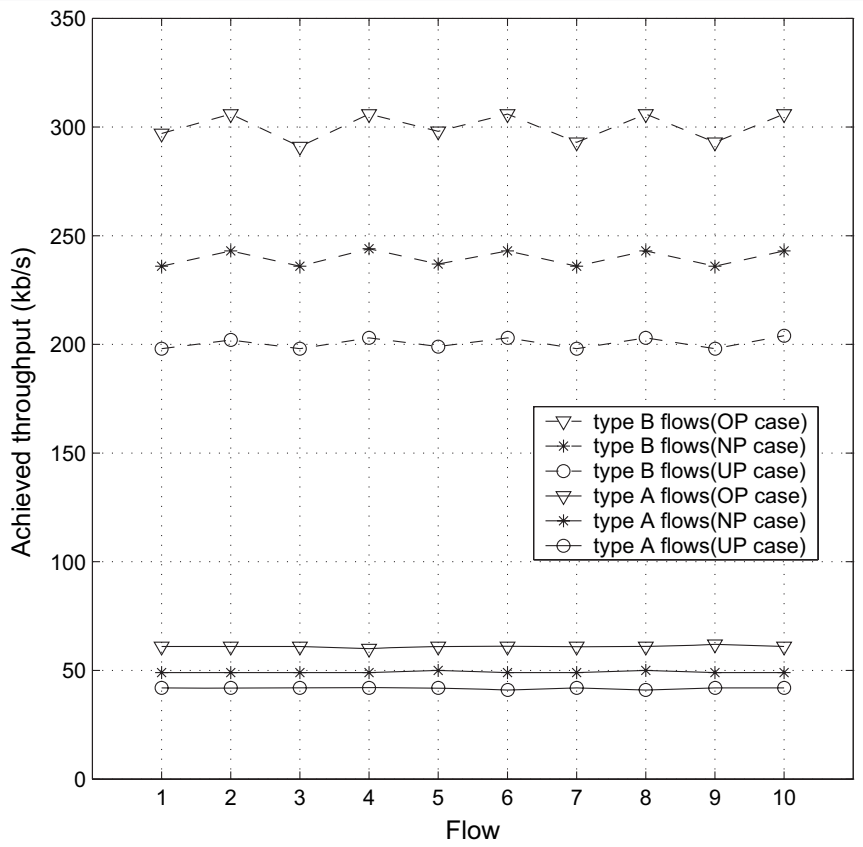

Fig. 10. Achieved throughput for data flows in the under-provisioned, nearprovisioned, and over-provisioned cases.

\section{Computation Complexity and System Overhead}

In the cross-layer design approach, the exhaustive search for the optimal design point for TCP/UDP traffic may be time-consuming. An effective solution is to generate a lookup table for different typical parameter values, thus avoiding computation burden in call duration. For the proposed MAC scheduler, some information needs to be exchanged between the BS and the MSs as follows. For voice service, the MS only needs to inform the BS of the instant of a new talk spurt or a new silence duration. For data flow $i$, as the short-term fairness weight is needed at the $\mathrm{BS}, W_{i}$ and $\tau_{s, i}$ should be updated to the BS upon a TCP packet arrival at the transmission queue in the MS, as both of them are updated at this rate at the MS. In addition, the BS needs to know if there are enough LL units in the transmission queue. If the LL unit number in this queue is less than $2 M_{i}$ (the upper bound of $m_{i}^{*}$ ) after next frame's transmission, the MS should inform the BS of this number; otherwise, the BS assumes that there are at least $2 M_{i}$ LL units to transmit for flow $i$. All the update information is transmitted in the reverse link request slot, using a more powerful channel coding technique to avoid transmission error, or piggybacked at the end of the transmitted LL units (if any) to reduce contention in the request slot. It can be seen that per-flow information is exchanged, kept, and used in the MAC packet scheduler. For DiffServ edge router (i.e., the BS), perflow information and processing does not induce scalability problem [18].

In the MAC scheduler, the per-frame scheduling is used, because the traffic arrivals are dynamic and per-frame scheduling can efficiently utilize the scarce wireless resources. In the literature, per-frame scheduling has shown its potential in CDMA MAC schedulers [5], [6]. In addition, the performance of schedulers in [5], [6] depends on a good estimate of the system capacity (i.e., how many flows from different traffic classes can be admitted into the system without violation of their QoS requirements). Unfortunately, no analytical admission region is given. On the other hand, for our cross-layer approach, an implicit admission region is that the sum of the equivalent resource amounts of all the flows is not greater than the available resource amount in the system. The cross-layer design provides a more efficient solution for QoS provisioning.

\section{E. Energy Efficiency Issue}

In a wireless mobile environment, each MS experiences path loss, shadowing, fading, and interference. In this research, to achieve a target bit energy to interference-plus-noise density ratio, perfect power control is assumed for the CDMA uplink transmission. This means that, when the transmission link quality is poor, a high power level should be used, and sometimes a very high power level may not be affordable. This is not energy efficient for the power-supply-limited MS. In addition, larger transmission power in one cell leads to larger interference to neighboring cells, thus reducing the overall system capacity in a multi-cell environment. Therefore, it is desired to switch off MS transmission in the case of poor link quality (e.g., under a threshold), and switch it on when the channel quality gets better. This mechanism can be smoothly incorporated to the system model under consideration. The effect of the energy-efficiency mechanism is an extra transmission delay induced by the idle time of the transmitter, which is determined by the transmission environment, user mobility, and the link quality threshold. For data traffic, the extra delay can be taken into account by $D_{w e}$, while for voice traffic, a more stringent wireless delay bound should be applied to deal with the extra delay.

\section{CONCLUSION}

This paper explores the idea of a cross-layer design approach in wireless cellular networks to guarantee QoS in 
the high layer and achieve efficient resource utilization in the low layer. We propose a protocol stack design based on characteristics and QoS requirements of the different traffic classes. We find the optimal equivalent resource amounts for voice and data traffic with the constraint of transport layer QoS guarantee. Furthermore, we propose a MAC scheduling scheme (which uses per-flow information) for an integrated voice/data traffic environment to achieve efficient statistical multiplexing. With an appropriate call admission control strategy in place, our scheme provides a solution to interconnect wireless cellular networks with DiffServ Internet backbone.

So far we have considered QoS provisioning for voice and data traffic in a single-cell case. In a multi-cell environment, due to the inter-cell interference, the schedulers in different cells should not act independently, thus making the resource allocation much more complex. Many related issues (such as power assignment and user mobility pattern) need further investigation.

\section{ACKNOWLEDGEMENTS}

The authors would like to thank the Editor Dr. E. Hossain and the anonymous reviewers for their constructive comments which improve the presentation of this paper.

\section{REFERENCES}

[1] S. Blake, D. Black, M. Carlson, E. Davies, Z. Wang, and W. Weiss, "An architecture for differentiated services," IETF RFC 2475, Dec. 1998.

[2] T.-K. Liu and J. A. Silvester, "Joint admission/congestion control for wireless CDMA systems supporting integrated services," IEEE J. Select. Areas Commun., vol. 16, no. 6, pp. 845-857, Aug. 1998.

[3] H. Kang, D. Kim, C. Lee, and K. Kim, "A throughput-efficient code assignment scheme for an integrated voice/data multi-code CDMA system," in Proc. IEEE VTC'00, vol. 2, pp. 1494-1497, 2000.

[4] I. Koo, S. Bahng, and K. Kim, "QoS-sensitive admission policy for non-real-time data packets in voice/data integrated CDMA systems," in Proc. IEEE GLOBECOM'01, vol. 4, pp. 2611-2616, 2001.

[5] I. F. Akyildiz, D. A. Levine, and I. Joe, "A slotted CDMA protocol with BER scheduling for wireless multimedia networks," IEEE/ACM Trans. Networking, vol. 7, no. 2, pp. 146-158, Apr. 1999.

[6] V. Huang and W. Zhuang, "QoS-oriented packet scheduling for wireless multimedia CDMA communications," IEEE Trans. Mobile Computing, vol. 3, no. 1, pp. 73-85, Jan-Feb. 2004.

[7] V. Jacobson, K. Nichols, and K. Poduri, "An expedited forwarding PHB," IETF RFC 2598, June 1999.

[8] J. Heinanen, F. Baker, W. Weiss, and J. Wroclawski, "Assured forwarding PHB group," IETF RFC 2597, Jun. 1999.

[9] C.-L. I and R. D. Gitlin, "Multi-code CDMA wireless personal communications networks," in Proc. IEEE ICC'95, vol. 2, pp. 1060-1064, 1995.
[10] H. Balakrishnan, V. N. Padmanabhan, S. Seshan, and R. H. Katz, "A comparison of mechanisms for improving TCP performance over wireless links," IEEE/ACM Trans. Networking, vol. 5, no. 6, pp. 756769, Dec. 1997.

[11] S. Floyd and V. Jacobson, "Random early detection gateways for congestion avoidance," IEEE/ACM Trans. Networking, vol. 1, no. 4, pp. 397-413, Aug. 1993.

[12] A. Sampath, P. Sarath Kumar, and J. M. Holtzman, "Power control and resource management for a multimedia CDMA wireless system," in Proc. IEEE PIMRC'95, vol. 1, pp. 21-25, 1995.

[13] K. Nichols and B. Carpenter, "Definition of differentiated services per domain behaviors and rules for their specification," IETF RFC 3086, Apr. 2001.

[14] J. Padhye, V. Firoiu, D. F. Towsley, and J. F. Kurose, "Modeling TCP Reno performance: A simple model and its empirical validation," IEEE/ACM Trans. Networking, vol. 8, no. 2, pp. 133-145, Apr. 2000.

[15] H. M. Chaskar, T. V. Lakshman, and U. Madhow, "TCP over wireless with link level error control: analysis and design methodology," IEEE/ACM Trans. Networking, vol. 7, no. 5, pp. 605-615, Oct. 1999.

[16] S. Floyd, M. Handley, J. Padhye, and J. Widmer, "Equation-based congestion control for unicast applications," in Proc. ACM SIGCOMM'00, pp. 43-56, Aug. 2000.

[17] M. Baines et al., "Using TCP models to understand bandwidth assurance in a differentiated services network," in Proc. IEEE GLOBECOM'01, vol. 3, pp. 1800-1805, Nov. 2001.

[18] X. Xiao and L.M. Ni, "Internet QoS: a big picture," IEEE Network, vol. 13, no. 2, pp. 8-18, Mar-Apr. 1999.

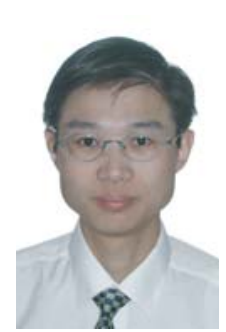

Hai Jiang (S'04) received the B.S. degree in 1995 and the M.S. degree in 1998, both in electronics engineering, from Peking University, Beijing, China. $\mathrm{He}$ is currently working toward his Ph.D. degree at the Department of Electrical and Computer Engineering, University of Waterloo, Ontario, Canada. His current research interests include quality-ofservice provisioning and resource management for multimedia communications in all-IP wireless networks.

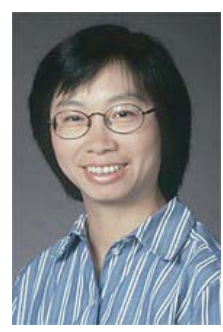

Weihua Zhuang (M'93-SM'01) received the B.Sc. and M.Sc. degrees from Dalian Maritime University, Liaoning, China, and the Ph.D. degree from the University of New Brunswick, Fredericton, NB, Canada, all in electrical engineering. Since October 1993, she has been with the Department of Electrical and Computer Engineering, University of Waterloo, ON, Canada, where she is a full professor. She is a co-author of the textbook Wireless Communications and Networking (Prentice Hall, 2003). Her current research interests include multimedia wireless communications, wireless networks, and radio positioning. Dr. Zhuang is a licensed Professional Engineer in the Province of Ontario, Canada. She received the Premier's Research Excellence Award (PREA) in 2001 from the Ontario Government for demonstrated excellence of scientific and academic contributions. She is an Associate Editor of IEEE Transactions on Wireless Communications, IEEE Transactions on Vehicular Technology, and EURASIP Journal on Wireless Communications and Networking. 